\title{
Nonperturbative calculations of form factors for exclusive semileptonic $B_{(s)}$ decays
}

\section{Jonathan M. Flynn, ${ }^{a}$ Ryan C. Hill, ${ }^{a, b}$ Andreas Jüttner, ${ }^{a}$ Amarjit Soni, ${ }^{c}$ J. Tobias Tsang $^{d}$ and Oliver Witzel ${ }^{e, f, *}$}

${ }^{a}$ Physics and Astronomy, University of Southampton, Southampton SO17 1BJ, UK

${ }^{b}$ DISCnet Centre for Doctoral Training, University of Southampton, Southampton SO17 1BJ, UK

${ }^{c}$ Physics Department, Brookhaven National Laboratory, Upton, NY 11973, USA

${ }^{d}$ CP3-Origins and IMADA, University of Southern Denmark, Campusvej 55, DK-5230 Odense M, Denmark

${ }^{e}$ Department of Physics, University of Colorado, Boulder, CO 80309, United States

${ }^{f}$ Theoretische Physik 1, Naturwissenschaftlich-Technische Fakultät, Universität Siegen, 57068 Siegen, Germany

E-mail: r.c.hill@soton.ac.uk, oliver.witzel@uni-siegen.de

Precise theoretical predictions derived from the Standard Model are a key ingredient in searches for new physics in the flavor sector. The large mass and long lifetime of the $b$ quark make processes involving $b$ quarks of particular interest. We use lattice simulations to perform nonperturbative QCD calculations for semileptonic $B_{(s)}$ decays. We present results from our determinations of $B_{s} \rightarrow D_{s} \ell v$ and $B_{s} \rightarrow K \ell v$ semileptonic form factors and provide an outlook for our $B \rightarrow \pi \ell v$ calculation. In addition we discuss the determination of $R$-ratios testing lepton-flavor universality and suggest use of an improved ratio. Our calculations are based on the set of $2+1$ flavor domain wall Iwasaki gauge field configurations generated by the RBC-UKQCD collaboration featuring three lattice spacings of $1 / a=1.78,2.38$, and $2.79 \mathrm{GeV}$. Heavy $b$-quarks are simulated using the relativistic heavy quark action.

For the RBC and UKQCD collaborations

40th International Conference on High Energy physics - ICHEP2020

July 28 - August 6, 2020

Prague, Czech Republic (virtual meeting)

${ }^{*}$ Speaker 


\section{Introduction}

Semileptonic decays of $B_{(s)}$ mesons play an important role in testing and constraining the Standard Model (SM) of elementary particle physics. Theoretical calculations of such processes, in combination with experimental data, allow extraction of Cabibbo-Kobayashi-Maskawa (CKM) matrix elements, or comparison of theory and experiment for ratios testing lepton flavor universality. Focusing on exclusive semileptonic decays, we use lattice quantum chromodynamics (QCD) simulations to obtain from first principles the form factors parametrizing the contributions due to the strong force. Specifically we report on our work for $B_{s} \rightarrow D_{s} \ell v$ and $B_{s} \rightarrow K \ell v$ decays and in addition present an outlook on our calculation of $B \rightarrow \pi \ell v$ decays. Each of these processes can be described by two form factors, $f_{+}$and $f_{0}$, which parametrize the semileptonic decay rate

$$
\begin{aligned}
\frac{d \Gamma\left(B_{(s) \rightarrow P \ell v)}\right.}{d q^{2}}=\eta_{\mathrm{EW}} \frac{G_{F}^{2}\left|V_{x b}\right|^{2}}{24 \pi^{3}} \frac{\left(q^{2}-m_{\ell}^{2}\right)^{2}|\vec{k}|}{\left(q^{2}\right)^{2}}\left[\left(1+\frac{m_{\ell}^{2}}{2 q^{2}}\right) \vec{k}^{2}\left|f_{+}\left(q^{2}\right)\right|^{2}\right. & \\
& \left.+\frac{3 m_{\ell}^{2}}{8 q^{2}} \frac{\left(M_{B_{(s)}}^{2}-M_{P}^{2}\right)^{2}}{M_{B_{(s)}}^{2}}\left|f_{0}\left(q^{2}\right)\right|^{2}\right]
\end{aligned}
$$

where $x=c$ for $P=D_{s}$ and $x=u$ for $P=K, \pi$. The corresponding meson masses are denoted by $M_{B_{(s)}}$ and $M_{P}$ and the 4-momenta by $p$ and $k$, respectively. $E$ labels the energy of the $P$-meson and $q=p-k$ is the momentum transfer with $|\vec{k}|=\left(E^{2}-M_{P}^{2}\right)^{1 / 2} . G_{F}$ is the Fermi constant and $\eta_{\mathrm{EW}}$ an electroweak correction factor. After presenting our form factors for the three different processes, we discuss testing lepton flavor universality by defining $R$-ratios of form factors integrated over different ranges in $q^{2}$.

Our numerical work is based on using RBC-UKQCD's set of $2+1$ flavor gauge field configurations [1-4] featuring the physical effects of two degenerate up/down quarks as well as the strange quark in the sea sector and inverse lattice spacings of $1.78,2.38$, and $2.79 \mathrm{GeV}$. Light, strange, and charm quarks are simulated using a domain-wall action [5-10], whereas bottom quarks are simulated using the relativistic heavy quark action [11-13]. In total we use six different ensembles with pion masses down to $267 \mathrm{MeV}$.

\section{Semileptonic $B_{s} \rightarrow D_{s} \ell v$ and $B_{s} \rightarrow K \ell v$ decays}

The LHCb collaboration recently presented the first experimental results for exclusive semileptonic $B_{S}$ decays [14, 15]. With improved statistical precision and a finer resolution of the $q^{2}$ bins, $B_{s}$ decays will provide an interesting, alternative channel to determine the CKM matrix elements $\left|V_{c b}\right|$ and $\left|V_{u b}\right|$ which so far are extracted using $B \rightarrow D^{(*)} \ell v$ and $B \rightarrow \pi \ell v$ decays, respectively. Eventually $B_{S}$ decays may help shed light on the persistent discrepancy between inclusive and exclusive determinations [16] or help address the tension with the SM observed in the ratio $R_{D^{(*)}}$ testing lepton flavor universality. The nonperturbative lattice calculation favors $B_{s}$ decays over $B$ decays because both numerical costs and statistical uncertainties grow with decreasing light-quark mass. Calculations for $B_{S}$ mesons are therefore computationally cheaper with smaller statistical errors than comparable calculations for $B$ mesons. In addition the larger mass of the hadronic final state in the $B_{s}$ decay reduces the amount of energy released, which makes the calculation slightly easier. 

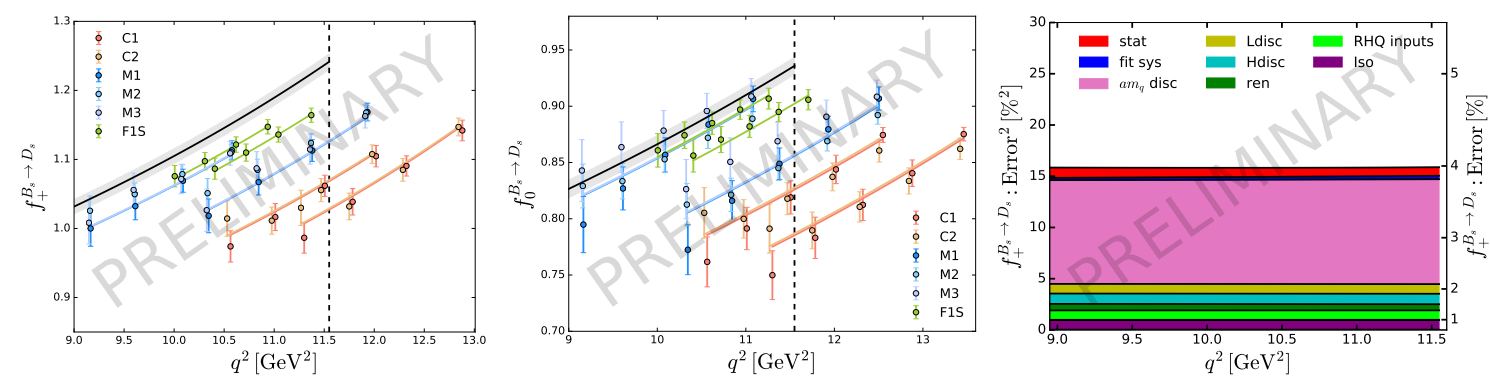

Figure 1: Form factors $f_{+}$and $f_{0}$ for semileptonic $B_{s} \rightarrow D_{s} \ell v$ decays. The left and central panels show the outcome of our numerical simulations as well as the chiral-continuum limit obtained from a combined and correlated fit to all data points. The right panel presents our full error budget for $f_{+}$which is presently dominated by the uncertainty due to discretizing the charm quarks.
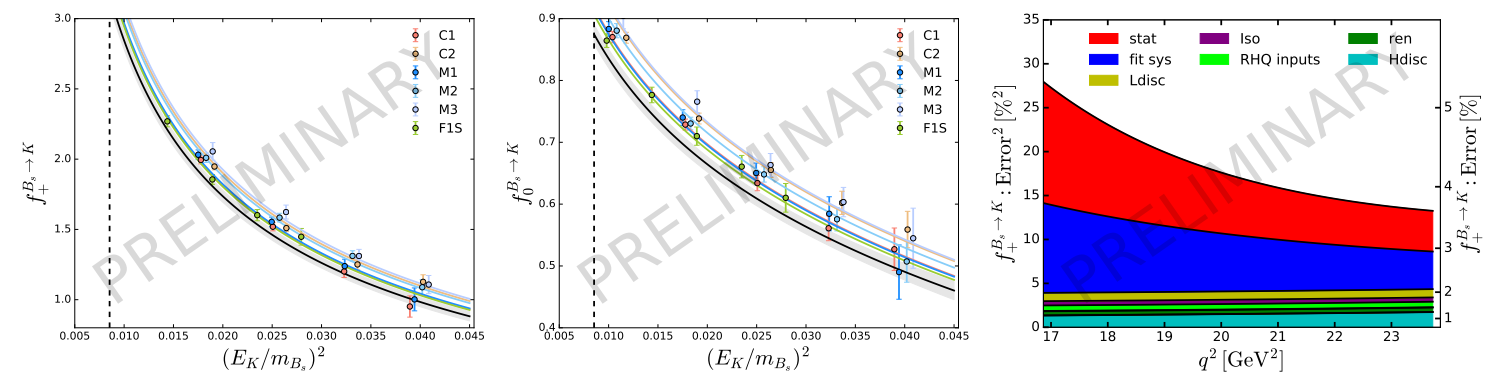

Figure 2: Form factors $f_{+}$and $f_{0}$ for semileptonic $B_{s} \rightarrow K \ell v$ decays (left and central panel) and full error budget for $f_{+}$(right panel) where at present effects due to statistical uncertainties dominate.

We present our results for the $f_{+}$and $f_{0}$ form factors for $B_{s} \rightarrow D_{s} \ell v$ decays in the left and central panels of Fig. 1. The colored data points are the outcome of our numerical simulations on the six different gauge field ensembles and the color indicates the lattice spacing: in orange coarse ensembles $(\mathrm{C})$, in blue medium ensembles $(\mathrm{M})$, and in green the fine ensemble (F). Next we perform a combined and correlated fit of all data points to obtain the chiral-continuum limit (black line with gray error band), i.e. the form factor at physical quark masses in the continuum for the range of $q^{2}$ we directly access in our simulation. This global fit is based on a polynomial ansatz using a low order Padé approximation. The right panel gives a preview of our full error budget where in addition to the statistical uncertainty (red), we consider variations to the fit function (blue) and estimate further systematic effects. At present the uncertainty due to the discretization of the charm quark (magenta) dominates the error budget. While we show the error budget only for $f_{+}$, that for $f_{0}$ looks similar.

In Fig. 2 we similarly show the status of our analysis for $B_{s} \rightarrow K \ell v$. The left and central panels again show the form factors as we calculate them on the lattice in combination with the result in the chiral-continuum limit. For $B_{s} \rightarrow K \ell v$ we obtain the latter by performing a global fit to all data points using an ansatz inspired by $\mathrm{SU}(2)$ heavy meson chiral perturbation theory (HM $\chi \mathrm{PT})$ in the limit for hard kaons. As the panel on the right indicates, our results are at present dominated by the statistical uncertainties.

With the full results of lattice calculation for $B_{s} \rightarrow D_{s} \ell v$ and $B_{s} \rightarrow K \ell v$ form factors to 

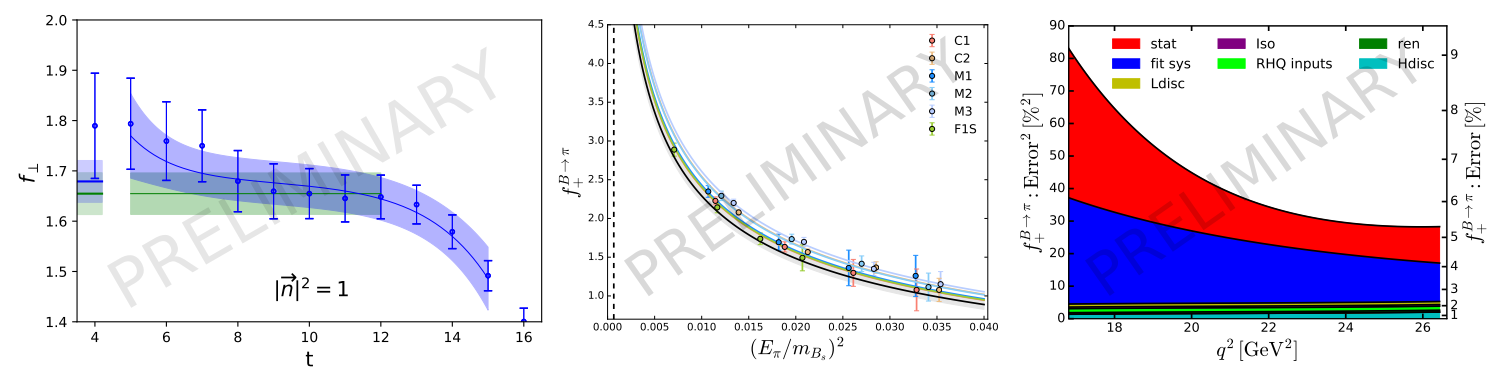

Figure 3: View of our $B \rightarrow \pi \ell v$ calculation. Left: comparison between ground state and excited state fit to extract the form factor signal with one unit of momentum on the coarse $\mathrm{C} 1$ ensemble. Center: Lattice determination of the form factor $f_{+}$as well as the chiral-continuum limit obtained from an $\mathrm{SU}(2) \mathrm{HM} \chi \mathrm{PT}$ fit. Right: preliminary error budget for $f_{+}$.

hand, we are currently studying the kinematical extrapolation of our form factors over the full range of $q^{2}$ using the $z$ parametrization following Boyd, Grinstein and Lebed (BGL) [17] or Bourrely, Caprini and Lellouch (BCL) [18]. Scrutinizing the $z$ expansion for possible systematic effects, for example due to the order of the expansion, is important in order to obtain reliable predictions for ratios testing lepton flavor universality since these are computed by integrating the decay rate over physically allowed regions in $q^{2}$.

\section{Semileptonic $B \rightarrow \pi \ell v$ decays}

In parallel we are working on an update of our $B \rightarrow \pi \ell v$ calculation from 2015 [19]. Including the new fine ensemble (F1S) at a third, finer lattice spacing combined with improved estimates for systematic effects and an enhanced analysis, we intend to obtain an improved result with reduced uncertainties. In the first step of the analysis, we refine the extraction of the form factors by including additional terms parametrizing one excited state for both the $B$ meson as well as the pion. This leads to a larger range of time slices entering the determination and hence a better estimate as can be seen in the left most plot of Fig. 3 where a simple groundstate fit (green) is compared to the one including excited states (blue). Extracting values for the form factors on all six ensembles and using up to four units of momenta, we show in the central panel our values to determine $f_{+}$combined with the chiral-continuum limit obtained using $\mathrm{SU}(2) \mathrm{HM} \chi \mathrm{PT}$. A preview of the corresponding full error budget is shown in the right most panel of Fig. 3 where again effects due to statistical uncertainties dominate.

\section{Ratios testing lepton flavor universality violations}

Lepton flavor universality is an accidental symmetry in the SM. One way to test it is to consider semileptonic decays with different leptonic $(e, \mu, \tau)$ final states. Although $e, \mu$ and $\tau$ have the same couplings to $W$ and $Z$ bosons in the SM, their different masses mean that the shapes of differential decay widths with respect to $q^{2}$ differ, as do (partially) integrated decay rates. A commonlyperformed test of the SM is to compare experimentally measured and theoretically predicted values 
for the ratio

$$
R_{D_{s}^{(*)}}=\int_{m_{\tau}^{2}}^{q_{\max }^{2}} d q^{2} \frac{d \Gamma\left(B_{s} \rightarrow D_{s}^{(*)} \tau \bar{v}_{\tau}\right)}{d q^{2}} \mid \int_{m_{l}^{2}}^{q_{\max }^{2}} d q^{2} \frac{d \Gamma\left(B_{s} \rightarrow D_{s}^{(*)} l \bar{v}_{l}\right)}{d q^{2}},
$$

where the integration is taken from $q^{2}=m_{l(\tau)}^{2}$ to the maximum value of $q^{2}$ that is kinematically allowed, and $l$ denotes either $e$ or $\mu$.

This definition of $R_{D_{s}^{(*)}}$ has the drawback that the denominator contribution from $m_{l}^{2} \leq q^{2} \leq m_{\tau}^{2}$ has no correspondence in the numerator and hence cannot provide useful information to test lepton flavor universality. We therefore follow $[20,21]$ and consider a different ratio

$$
R_{D_{s}^{(*)}}^{\mathrm{imp}}=\int_{q_{\min }^{2}}^{q_{\max }^{2}} d q^{2} \frac{d \Gamma\left(B_{s} \rightarrow D_{s}^{(*)} \tau \bar{v}_{\tau}\right)}{d q^{2}} \mid \int_{q_{\min }^{2}}^{q_{\max }^{2}} d q^{2}\left[\frac{\omega_{\tau}\left(q^{2}\right)}{\omega_{l}\left(q^{2}\right)}\right] \frac{d \Gamma\left(B_{s} \rightarrow D_{s}^{(*)} l \bar{v}_{l}\right)}{d q^{2}}
$$

using a common integration range in the numerator and denominator with lower limit $q_{\min }^{2} \geq m_{\tau}^{2}$, and reweighting the integrand in the denominator with the factor $\omega_{\tau}\left(q^{2}\right) / \omega_{l}\left(q^{2}\right)$, where

$$
\omega_{\ell}\left(q^{2}\right)=\left(1-\frac{m_{\ell}^{2}}{q^{2}}\right)^{2}\left(1+\frac{m_{\ell}^{2}}{2 q^{2}}\right) \quad \text { for } \quad \ell=e, \mu, \tau
$$

This form of the ratio was introduced in [21] for hadronic vector final states and we adopt it for pseudoscalar final states such as for $B_{s} \rightarrow D_{s} \ell v$ decays. The contribution of the scalar form factor in the denominator of Eq. (3) can be neglected because $m_{\mu}^{2} /\left(m_{\mu}^{2}+2 q^{2}\right) \ll 1$ for $q^{2} \in\left[m_{\tau}^{2}, q_{\max }^{2}\right)$ and we use the decay rate expression from Eq. (1) to find

$$
R_{D_{s}}^{\mathrm{imp}, \mathrm{SM}}=1+\frac{\int_{q_{\min }^{2}}^{q_{\max }^{2}} d q^{2} \Phi\left(q^{2}\right) \omega_{\tau}\left(q^{2}\right)\left(F_{S}^{\tau}\right)^{2}}{\int_{q_{\min }^{2}}^{q_{\max }^{2}} d q^{2} \Phi\left(q^{2}\right) \omega_{\tau}\left(q^{2}\right) F_{V}^{2}}
$$

with $\Phi\left(q^{2}\right)=\eta_{\mathrm{EW}} G_{F}^{2}\left|V_{x b}\right|^{2} \vec{k} / 24 \pi^{3}$ and

$$
\begin{aligned}
\left(F_{S}^{\tau}\right)^{2} & =\frac{3}{4} \frac{m_{\tau}^{2}}{m_{\tau}^{2}+2 q^{2}} \frac{\left(M^{2}-m_{\tau}^{2}\right)^{2}}{M^{2}}\left|f_{0}\left(q^{2}\right)\right|^{2} \\
F_{V}^{2} & =\vec{k}^{2}\left|f_{+}\left(q^{2}\right)\right|^{2}
\end{aligned}
$$

\section{Acknowledgments}

We thank our RBC and UKQCD collaborators for helpful discussions and suggestions. Computations used resources provided by the USQCD Collaboration, funded by the Office of Science of the US Department of Energy and by the ARCHER UK National Supercomputing Service, as well as computers at Columbia University and Brookhaven National Laboratory. We used gauge field configurations generated on the DiRAC Blue Gene Q system at the University of Edinburgh, part of the DiRAC Facility, funded by BIS National E-infrastructure grant ST/K000411/1 and STFC grants ST/H008845/1, ST/K005804/1 and ST/K005790/1. This project has received funding from Marie Skłodowska-Curie grant 659322 (EU Horizon 2020), STFC grants ST/P000711/1 and 
ST/T000775/1. RH was supported by the DISCnet Centre for Doctoral Training (STFC grant ST/P006760/1). OW acknowledges support from DOE grant DE-SC0010005. AS was supported in part by US DOE contract DE-SC0012704. JTT acknowledges suppport from the Independent Research Fund Denmark, Research Project 1, grant 8021-00122. No new experimental data was generated. The project leading to this application has received funding from the European Union's Horizon 2020 research and innovation programme under the Marie Skłodowska-Curie grant agreement No 894103.

\section{References}

[1] RBC/UKQCD collaboration, C. Allton et al., Phys. Rev. D78 (2008) 114509 [0804 . 0473].

[2] RBC/UKQCD collaboration, Y. Aoki et al., Phys.Rev. D83 (2011) 074508 [1011.0892].

[3] RBC/UKQCD collaboration, T. Blum et al., Phys. Rev. D93 (2016) 074505 [1411. 7017].

[4] RBC/UKQCD collaboration, P. A. Boyle, L. Del Debbio, A. Jüttner et al., JHEP 12 (2017) 008 [1701.02644].

[5] D. B. Kaplan, Phys. Lett. B288 (1992) 342 [hep-lat/9206013].

[6] Y. Shamir, Nucl. Phys. B406 (1993) 90 [hep-lat/9303005].

[7] V. Furman and Y. Shamir, Nucl. Phys. B439 (1995) 54 [hep-lat/9405004].

[8] T. Blum and A. Soni, Phys. Rev. D56 (1997) 174 [hep-lat/9611030].

[9] T. Blum and A. Soni, Phys. Rev. Lett. 79 (1997) 3595 [hep-lat/9706023].

[10] R. C. Brower, H. Neff and K. Orginos, Comput. Phys. Commun. 220 (2017) 1 [1206. 5214].

[11] N. H. Christ, M. Li and H.-W. Lin, Phys.Rev. D76 (2007) 074505 [hep-lat/0608006].

[12] H.-W. Lin and N. Christ, Phys.Rev. D76 (2007) 074506 [hep-lat/0608005].

[13] RBC/UKQCD collaboration, Y. Aoki, N. H. Christ, J. M. Flynn et al., Phys. Rev. D86 (2012) 116003 [1206.2554].

[14] LHCв collaboration, R. Aaij et al., Phys. Rev. D 101 (2020) 072004 [2001. 03225].

[15] LHCв collaboration, R. Aaij et al., 2003.08453.

[16] P. Gambino, M. Jung and S. Schacht, Phys. Lett. B795 (2019) 386 [1905 . 08209].

[17] C. G. Boyd, B. Grinstein and R. F. Lebed, Phys.Rev.Lett. 74 (1995) 4603 [hep-ph/9412324].

[18] I. Caprini, L. Lellouch and M. Neubert, Nucl. Phys. B530 (1998) 153 [hep-ph/9712417].

[19] RBC/UKQCD collaboration, J. M. Flynn, T. Izubuchi, T. Kawanai et al., Phys. Rev. D91 (2015) 074510 [1501.05373].

[20] D. Atwood and A. Soni, Phys. Rev. D 45 (1992) 2405.

[21] G. Isidori and O. Sumensari, Eur. Phys. J. C 80 (2020) 1078 [2007 . 08481]. 